\title{
Analysis of promoters and CREB/AP-1 binding sites of the human TMEM174 gene
}

\author{
FEN HU ${ }^{1 *}$, YANAN MENG ${ }^{1,2^{*}}$, LIXIA GOU $^{1}$ and XIUJUN ZHANG ${ }^{1}$ \\ ${ }^{1}$ College of Life Sciences, Hebei United University, Tangshan, Hebei 063000; \\ ${ }^{2}$ Department of Clinical Laboratory, Baoding People's Hospital, Baoding, Hebei 071051, P.R. China
}

Received April 14,2013; Accepted August 1, 2013

DOI: $10.3892 /$ etm.2013.1275

\begin{abstract}
Transmembrane protein 174(TMEM174) is a type III transmembrane protein with no clear signal peptide. The $\mathrm{N}$ and $\mathrm{C}$ terminals are located inside the cell. Our previous study demonstrated high expression of TMEM174 in the kidney and its potential involvement in renal cancer based on its capacity to stimulate cell proliferation. However, the mechanism by which TMEM174 promotes proliferation at the transcriptional level remains to be elucidated. In the present study, the TMEM174 promoter region was amplified from whole blood DNA. Six different regions of the regulatory sequences of the TMEM174 promoter region including $\sim 2.5 \mathrm{~kb}$ of the upstream region were cloned into the dual luciferase expression vector pGL3-basic. Comparison of the activity of these fragments in dual luciferase reporter assays revealed higher levels of activity for the fragments spanning -186 to $+674,-700$ to $+674,-1,000$ to +674 and $-2,500$ to $+1 \mathrm{bp}$. Lower levels of activity were detected for the fragments spanning -466 to +674 and -890 to $+674 \mathrm{bp}$. The highest activity was detected for the fragment spanning -186 to +674 bp. Electrophoretic mobility shift assay (EMSA) was performed to determine effective transcription factor binding sites. Specific binding of the cyclic-AMP response element binding (CREB) within the TMEM174 gene promoter region was demonstrated, although binding of the activator protein-1 (AP-1) was also detected in this region. In conclusion, these results suggest that the core promoter region of the human TMEM174 gene is located within the region spanning -186 to +674 bp and that the transcription factors CREB and AP-1 are involved in the transcriptional regulation of this gene.
\end{abstract}

Correspondence to: Dr Xiujun Zhang, College of Life Sciences, Hebei United University, 57 Jianshenan Road, Tangshan, Hebei 063000, P.R. China

E-mail: xiujunzhang66@126.com

*Joint senior authorship

Key words: activator protein-1, core promoter, cyclic-AMP response element binding protein, transmembrane protein 174 gene, transcriptional regulation, luciferase

\section{Introduction}

Transmembrane protein 174 (TMEM174) was identified among a large pool of genes by high-throughput cell screening technology, which is used to isolate functional genes and to provide insight into the mechanisms of gene function (1). Genes are investigated using a reverse biological strategy in which gene expression profiling of tissues and cell lines is performed. This approach revealed high expression levels of TMEM174 in kidney tissue and the lymphadenoma-derived Raji cell line (2). The TMEM174 gene is activated by the activator protein-1 (AP-1) pathway in HeLa and 293T cells ( 3- and 9-fold, respectively). Preliminary studies of the mechanism underlying the promotion of cell proliferation indicated that TMEM174 is linked with Ras and Raf in the extracellular-signal-regulated kinase (ERK) pathway. Furthermore, TMEM174 has a role in promoting the $\mathrm{G} 2 / \mathrm{S}$ phase of the cell cycle (2). However, the underlying mechanism remains to be elucidated and other pathways and transcription factors have been suggested to be involved in this process.

The cyclic-AMP response element binding (CREB) protein is a transcription factor that affects a spectrum of cellular activities. including glucose homeostasis, growth factor-dependent survival, proliferation, differentiation and memory $(3,4)$. CREB recruitment to the cyclin D1 promoter promotes cyclin D1 transcription and cell proliferation (5). Transgenic animal studies have shown that overexpression of CREB confers oncogenic characteristics on cells in various tissues and that abnormal CREB expression is associated with tumor development in humans (6). CREB plays an important role in the development of brain tumors, leukemias and other types of cancer (7). Evidence based on the high level of homology of zebrafish CREB and its mammalian counterpart suggests that activated (phosphorylated) CREB is localized in proliferation zones (7). Activation of CREB has been suggested to stimulate cellular proliferation through the PI3-K/AKT pathway (8) and to act as a crucial transcription factor for the regulation of TMEM174 expression.

AP-1 family members are important upstream activators of the ERK signaling pathway (9), which are involved in cell proliferation, transformation, differentiation and death (10-12). AP-1 is a collective term referring to dimeric transcription factors composed of Jun, Fos, activating transcription factor (ATF) and musculoaponeurotic fibrosarcoma (MAF) protein 
subunits that bind to a common AP-1 binding site $(13,14)$. c-Jun is a positive regulator of cell proliferation, while JunB mediates the converse effect. Jun promotes apoptosis by participating in cell stress-induced transcriptional activation of apoptotic target genes (15). Previous studies have indicated that TMEM174 overexpression activates AP-1, since the upstream molecules ERK, ELK-1 and Fos are markedly activated or stimulated by sequential blockade of these upstream factors in the ERK pathway (2).

These studies contribute to the understanding of the mechanism by which cell proliferation is promoted at the protein level. However, details of the mechanism at the transcriptional level remain to be elucidated. Identification of the core promoter is a critical first step in this process, facilitating subsequent determination of the important transcription factors.

In the present study, the mechanism by which TMEM174 promotes cell proliferation at the transcriptional level was investigated. The promoter region and transcription factor binding sites were predicted by bioinformatics analysis. Among the large numbers of binding sites predicted, such as CREB, AP-1, nuclear factor- $\kappa \mathrm{B}(\mathrm{NF}-\kappa \mathrm{B})$ and Oct1, CREB and AP-1 were identified with potential binding sites for interaction with the TMEM174 promoter region by electrophoretic mobility shift assay (EMSA).

\section{Materials and methods}

PCR amplification and molecular cloning. Primers were designed for the amplification of fragments of various lengths corresponding to the $4.8 \mathrm{~kb}$ sequence upstream of the TMEM174 gene (available in the NCBI mapview database: http://www.ncbi.nlm.nih.gov/mapview) and using TFEARCH online software (http://www.cbrc.jp/research/db/TFSEARCH. html) to predict the candidate promoter region and transcription factor binding sites. Fragments were amplified for cloning from whole blood genomic DNA. The primers used are shown in Table I.

The PCR amplification reaction system (Table II) conditions were as follows: initial denaturation at $95^{\circ} \mathrm{C}$ for $5 \mathrm{~min}$; 30 cycles of $95^{\circ} \mathrm{C}$ for $30 \mathrm{sec} ; 55-60^{\circ} \mathrm{C}$ (depending on the primer pair used) for $30 \mathrm{sec}$ and $72^{\circ} \mathrm{C}$ for $1 \mathrm{~min}$ and final elongation at $72^{\circ} \mathrm{C}$ for $7 \mathrm{~min}$. The PCR products were purified by agarose gel electrophoresis (Sigma-Aldrich, Schnelldorf, Germany) and Vigorous purification kits (Vigorous Biotechnology, Beijng, China). The purified products were cloned into the KpnI and HindIII restriction enzyme sites of pGL3-basic (Promega, Madison, WI, USA). The recombined vectors were sequenced by SinoGenoMax Co., Ltd. (Beijing, China).

Transfection and dual luciferase reporter assay. 293T cells were seeded in 96 -well plates $\left(1 \times 10^{4} /\right.$ well) in complete Dulbecco's modified Eagle's medium (DMEM; HyClone, Logan, UT, USA) containing $10 \%$ fetal bovine serum (FBS) and were transfected the next day with Vigofect (Vigorous Biotechnology) according to the instructions provided by the manufacturer. A total of $104 \mathrm{ng}$ plasmid DNA/well was transfected, including $100 \mathrm{ng}$ recombinant pGL3 plasmid or pGL3-basic (empty vector control) and $4 \mathrm{ng}$ pRL-TK (Promega) containing the Renilla luciferase gene as an internal control. Transfections were performed in triplicate. The cells were lysed in standard 1X lysis buffer (Promega) for $30 \mathrm{~min}$ at room temperature (RT) and the cell lysates were assayed for both firefly and Renilla luciferase activity using the Dual-Luciferase Reporter assay kit (Promega) according to the instructions provided by the manufacturer. Fluorescence was detected using a GENius Pro microtiter plate reader (Tecan, Männedorf, Switzerland). Relative luciferase activity was determined by normalizing the activity of the firefly luciferase activity ( $\mathrm{F}$ value) against the Renilla luciferase activity ( $R$ value). Each experiment was performed at least three times. The $F / R$ value was calculated respectively and an average value was obtained as the recombined plasmid activity. pGL3basic was used as the negative control and its activity was defined as 1 . The activities of all other recombined plasmids were compared with the negative control.

EMSA. Nuclear extracts of HepG2 cells were prepared using Nuclear Extract kits (Active Motif, Carlsbad, CA, USA). Protein concentrations were determined using BCA protein assay kits (Vigorous Biotechnology). The oligonucleotide probes (CREB, AP-1 and competitive probes) used in EMSA were designed according to a computer-based search with the software Promoter Scan (ProScan version 1.7 suite of programs developed by Dr Dan Prestridge, http://wwwbimas.cit.nih.gov/molbio/proscan/). Experimental groups were as follows: CREB probe/nuclear extract, AP-1 probe/ nuclear extracts, CREB probe/nuclear extract + competitive probe and AP-1 probe/nuclear extract + competitive probe. Free probe was used as a negative control. Nuclear extracts $(5 \mu \mathrm{g})$ were incubated with $2 \mu \mathrm{l}(200 \mathrm{nM})$ biotin-labeled DNA probe and mixed with 10X binding buffer $(2 \mu \mathrm{l}), 50 \%$ glycerol $(1 \mu \mathrm{l}), \mathrm{MgCl}_{2}(1 \mu \mathrm{l})$, Poly (dI.dC) $(1 \mu \mathrm{l})$ and 1\% NP-40 $(1 \mu \mathrm{l})$ for $10 \mathrm{~min}$ at RT. Bound DNA complexes were separated by non-denaturing polyacrylamide gel (6.5\%) electrophoresis and transferred to a positively charged nylon membrane. Cross-linking was performed under ultraviolet (UV) light for $20 \mathrm{~min}$ and the membranes were probed with a streptavidin-horseradish peroxidase (HRP) conjugate and incubated with the chemiluminescent substrate for band detection. For competition experiments, unlabeled competitor oligonucleotides were pre-incubated (200-fold excess) with the labeled probe.

Statistical analysis. Differences between the levels of expression were analyzed by one-way ANOVA. $\mathrm{P}<0.05$ was considered to indicate a statistically significant difference.

\section{Results}

TMEM174 promoter cloning. The promoter region of TMEM174 was amplified from whole blood genomic DNA as described in Materials and methods. Five fragments of various lengths corresponding to the predicted promoter region and the first exon of TMEM174 were amplified: -186 to +674 , -466 to $+674,-700$ to $+674,-890$ to +674 and $-1,000$ to $+674 \mathrm{bp}$. Amplified products of 860, 1,140, 1,347, 1,564 and 1,674 bp were cloned into pGL3-basic. The cloned sequences were identical to the original genomic sequences shown in Fig. 1A. Numerous transcription factor binding sites were predicted 
Table I. Primer sequences.

\begin{tabular}{|c|c|c|}
\hline Primer & Primer sequences & Position (bp) \\
\hline $\mathrm{P} 1$ & $\begin{array}{ll}\text { F: } & \text { 5'-CGGGGTACCGTTTTGCAAGTCAATGACAAGCTGTCTC-3' } \\
\text { R: } & \text { 5'-CCCAAGCTTCTTTCACGGACGGTGGAAATCACAG-3' }\end{array}$ & -186 to +674 \\
\hline $\mathrm{P} 2$ & $\begin{array}{ll}\text { F: } & \text { 5'-CGGGGTACCCAGCCAATTTTTAAAATTTTTTGTAGAGATAGG-3' } \\
\text { R: } & \text { 5'-CCCAAGCTTCTTTCACGGACGGTGGAAATCACAG-3' }\end{array}$ & -466 to +674 \\
\hline P3 & $\begin{array}{l}\text { F: 5'-CGGGGTACCCAGGAGTCTAACCTGATTTACCTAGTGGTTC-3' } \\
\text { R: 5'-CCCAAGCTTCTTTCACGGACGGTGGAAATCACAG-3' }\end{array}$ & -700 to +674 \\
\hline $\mathrm{P} 4$ & $\begin{array}{l}\text { F: 5'-CGGGGTACCGTTTGGGGAGTAATTCCAGCTTTGGG-3' } \\
\text { R: 5'-CCCAAGCTTCTTTCACGGACGGTGGAAATCACAG-3' }\end{array}$ & -890 to +674 \\
\hline P5 & $\begin{array}{ll}\text { F: } & \text { 5'-CGGGGTACCGACACATGCTTCGGACCCTCCCTC-3' } \\
\text { R: } & \text { 5'-CCCAAGCTTCTTTCACGGACGGTGGAAATCACAG-3' }\end{array}$ & -1000 to +674 \\
\hline P6 & $\begin{array}{l}\text { F: 5'-CGGGGTACCACAGGGAGACTTCAAGGTGGGAGAAAGGAG-3' } \\
\text { R: } \\
\text { 5'-CCCAAGCTTCTTCTATAACTAATTTGGACCTGTGATTCCTTG-3' }\end{array}$ & -2500 to +1 \\
\hline
\end{tabular}

Restriction enzyme sites were KpnI and HindIII. F, forward; R, reverse. Underlining indicates restriction enzyme sites.

Table II. PCR amplification reaction system.

\begin{tabular}{|c|c|}
\hline Substance & Value $(\mu 1)$ \\
\hline $2.5 \mathrm{mM}$ dNTP mixture & 2 \\
\hline 10X Pyrobest ${ }^{\mathrm{TM}}$ buffer & 2.5 \\
\hline Template DNA & 0.1 \\
\hline Forward primer & 2.5 \\
\hline Reverse primer & 2.5 \\
\hline Pyrobest $^{\mathrm{TM}}$ DNA polymerase & 0.3 \\
\hline $\mathrm{ddH}_{2} \mathrm{O}$ & 15.1 \\
\hline Total & 25 \\
\hline
\end{tabular}

within the promoter region by TFSEARCH, including CREB, AP-1, P300, NF- $\kappa$ B and Oct1 (Fig. 1B).

Characterization of TMEM174 promoter activity. Dual luciferase reporter assays were performed to detect promoter activity. Analysis of the recombined plasmids following transfection into 293T cells indicated that fragments -186 to +674 , -700 to $+674,-1,000$ to +674 bp and $-2,500$ to +1 bp exhibited higher levels of activity compared with fragments -466 to +674 and -890 to $+674 \mathrm{bp}$, with the two highest levels of activity detected for fragments -186 to +674 and $-2,500$ to +1 bp. Significant differences between groups are indicated in Fig. 2 .

Identification of transcription factors binding to the human TMEM174 promoter region. The identification of transcription factor binding sites within the predicted promoter region is important in determining the mechanism of transcriptional activation of this gene. Numerous transcription factor binding sites were identified within the predicted promoter region. Among these, CREB and AP-1 were related to cell proliferation. EMSA was performed as described in Materials and methods to investigate transcription factor binding. These experiments demonstrated specific binding of CREB with the TMEM174 promoter. The AP-1 probe designed according to the predicted TMEM174 promoter AP-1 binding site bound to HepG2 nuclear extract; this binding was partially inhibited by the addition of a competitive probe, indicating that AP-1 binds non-specifically within the TMEM174 promoter region (Fig. 3).

\section{Discussion}

A previous study focused on the mechanism by which TMEM174 promotes cell proliferation at the protein level have indicated potential signal transduction pathways involved in this process (2). The present study aimed to further elucidate this mechanism at the transcriptional level. Investigations of the activity of the predicted promoter showed that fragments -186 to $+674,-700$ to $+674,-1,000$ to +674 and $-2,500$ to +1 bp exhibited higher levels of activity compared with fragments -466 to +674 and -890 to +674 bp. The fragment -186 to +674 bp exhibited the highest activity indicating that the core promoter region might be located within this region of the TMEM174 promoter. Furthermore, the variation detected in the levels of activity demonstrated the regulatory complexity of this promoter. Based on the results of these experiments, the regions spanning -186 to -466 and -700 to -890 bp are suggested to contain strong negative regulatory elements. These data indicate that the apparent changes in promoter activity are the result of complex interactions between different regulatory elements.

TMEM174 has been identified as a potential regulator of cell proliferation using high-throughput cell screening technology (data not shown). This method allows the rapid identification of functional genes from a large pool and provides insights into to the function of these genes. However, this method is limited in its application for subsequent studies of the mechanism of gene function due to the potential omission of the role of such proteins in other pathways.

RNA in situ hybridization analysis showed that TMEM174 is highly expressed in some types of renal cancer, such as 


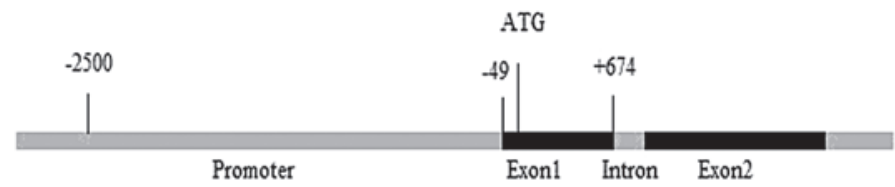

2501 CTCCTTTCTC CCACCTTCAAOTCTCCCTOT ACCACCCCCT ATIGCCAAR 2451 CCTACTCOACCACCCTCOSAUCSAAAAG TGTCCTTOA GAGTCTGAGA 2401 CAAAGAGAAG TITAGAAAOO TCCOTTTCOA OCTGAGTCAC CATACCTTAA

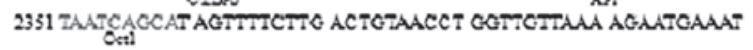
2301 TAIGTCACCT ATATTAHTE TATAGAATTA CACCTGCTAC ATACTAAGTA 2251 TTCAHTAAA CTTTCCCT CACTCATCAATTCACATGAT CGTCTOTTOT 2201 TTTTTCCCCT TCATTCTGTG AATATACTCC ATCACATAGA TTGATTTTTG 2151 IACGTTGAAC CACCCTTGCC TTCCACTTGG TCATGGCAIA TAGTCCTTTG 2101 AATATCCTAT TCAATTCAGT TCGTTACC TITA TAAAAHAA TTCCTATTAA 2051 CCATCTAGAC AGAGGTGCOC CTITTGGAG AAGCACCTGA GTCAAACGGA

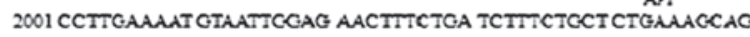
1951 TTCCAOTTTCCATTCATCAG CTOTCTAAHO CACCOTCATC TOTITCCTCT 1901 ACTCTCTCAO CCTTCCCAGT TATTCTIAGT COATATACCT AUACOCALA 1851 CCAAAACATA AATTGAGTCA TTIAGAAGAC AGTIITGAGA TCCTACCTT 1801 TTTCTAATC TCACCCATTE TTTTCACTGT TLCTTACOA GTAACAACC 1751 TGAAAATAAT CCCAGCCTAA CTIACCAGAA ATTTATAGTT AAMGAGAAAA 1701 TCTGCCTTIC CTGGATCTCT GATC TAGCTC AGTCCGAUA ACCCCAGCCT 1651 GTTCCTAIAG CAACTCCACT CCACCCTTCC TCCTCCAACS CACATCCAGO 1601 AAGATACCTC ACCCGTCACC TCCTTGCTTT TCCATCTOTT TGCCAGTCAA 1551 GTAGGAGAT ACAACCCTTC TCCTGTACCA GCSAGACCCA TITTCATCEO 1301 GAAAAATCTC CTTTCGTCTC CTACAAGCAT TAAAGGTIAA CCTCCCGTTO 1451 COTTECCTAA GATTCTTGA TOATCAATCA CCTGACOTCC TTCCCTTCOA 1401 TATTTCOTTC TOTTCOTCTO AAATCCOAAC TCACATTTTOTCC TTIAAAA 1351 AACTOOCCCATCTOATTCT TTOTCAACOO ANOTFAOCA AOCCCTCCCT 1301 TAGCOCAAGA CECCACCCCT CTCAATTCTE CAAAACCACC TAATATAATA 1251 TTLATTACT CСAGACACCA TCCCCTCAA TAAATCTCT CTACGACGA
1201 AAGAACCCTA CAGTTATAA TCAGTGGAGO AMGAAACTCA TCCCCCTGTT 1151 TCGAGGTT CCAGTCATCA ATCACAGATA ATGTLATTGA AMATIAAACT 1101 OTCTTCTCTACATCAACTTO COITTCOTIO TAAAACATAO TACOTTCCAA 1031 AACACACCTE GTOTTTCAC CTTCAAGACC ACOAACTOTO TCOCCACCAT 1001 GCACCGACCG TCCGAACCAT GTOTCCTAGT CACCCAGGTC ATATCTCATC 951 CTAATCAAC CACTCCCACT CATCCACTCC ATUUAACOT TICCTATCAC 901 GTTGTTCCTC TCCCAAAGT GGAATTACTC CCCAAACCCA GAAACCAAAA 851 GGCATGTGGG CCTGGCCAGA AHAGAACAGG ATCTCAGCCC TTTGTGAGAA SO1 CTCTTATTAAAGTTAAGTA TCATCACACC TACCCSTTCT TGAAGTAGAT 751 AGGATACAC ACGCAGTCAA GAAATACGTO TCTGTCTATT CCTAATGAC 701 TGAACCACTA GeThaATCAG OTTACACTCC TOTCCATGTG TGGATATATC 651 AHCOCCCCCATTCACTCCTC CACACCCTTO ACCCACCTCT CCCATTTCTA 601 CAHCTTCOE TACGACAMA ACACOCTCTT ATCOCACCOT CTTHATTCC 551 TATAGAAGAO TACCTCCTCO CCCCAACCCA OCACGACCAC TTCAGTCCAO

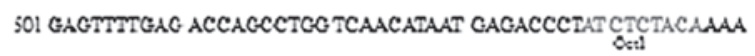
451 AATTTTAAAAATTCECTCAG CATTGTGATG TOTCCCTGTA GTCCTCECTA 401 CTCGAGAGGA TGACGTCCGA GGATCCCTTG ACCCAGGAGG TTCAGCCTCC 351 AGTGAGCTAT CATTGTCCCA CTCCATTCCACCTCCOTGAC AGAGTGAGAC

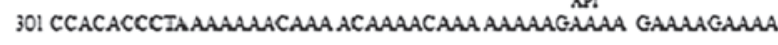
251 AGAMAGAGAA AGATCCOCTC AMTAMGTTAA TTITTGANTG TGTCAATCAA 201 TOAATGTGAA TTAGAGAGAC ACCTTOTCAT TGACTTCCAAAACAGACACC 151 TCCACTTOT GACANACAT GACAHACTT CACCCTTCAT TTCTCACACC 101 TTIATTCTIC TAAACAACOT CCACCCCTCT OTCTOTTACA CCCTOTTAAO S1CACACCCCTAAGTTCTTCT ATACTAUTT TCOACCTCTO ATTCCTTCOT 1 TCTCACAATC CTCTCCACTC TAACAACCAG COTGACCCCACAACCACCA

Figure 1. (A) Promoter region of the TMEM174 gene according to the Ensembl genome database. TMEM174 consists of 732 bp encoding putative 243 amino acids containing 2 exons. In the present study, the promoter region and the first exon were cloned into pGL3-basic. (B) Sequence of part of the promoter region showing potential transcription factor binding sites predicted by TFSEARCH (C/EBPb, AP-1, Oct1, CREB, STRE, c-Myb, P300). Selected transcription factors are shown. Nucleotides are numbered from the start codon ATG. AP-1, activator protein-1; CREB; cyclic-AMP response element binding.
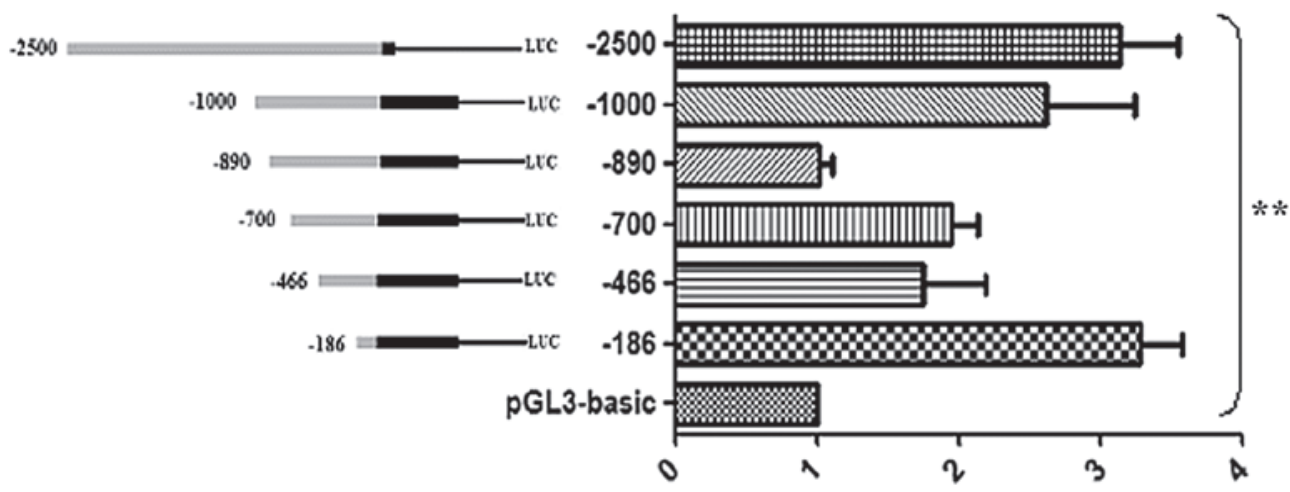

Figure 2. Dual luciferase reporter gene assays of TMEM174 gene promoter constructs. Various recombinant vectors and pRL-TK were co-transfected into 293 T cells. pRL-TK and pGL3-basic were used as internal and negative controls, respectively. Relative luciferase activity was determined by normalizing the activity of firefly luciferase against Renilla luciferase activity. Data represent the mean $\pm \mathrm{SD}$ of three independent experiments. * $\mathrm{P}<0.05$. The $\mathrm{P}$-value was calculated using one-way ANOVA that compared the activities of the 7 plasmids.

squamous cell carcinoma with necrosis, papillary renal cell carcinoma and transitional cell carcinoma, and that TMEM174 is expressed in the majority of renal cancers and pyelonephritis (data not shown). These data indicate that TMEM174 plays a role in the development of renal cancer.
NF- $\kappa$ B, STAT3, AP-1, CREB and nuclear factor erythroid 2-related factor ( $\mathrm{Nrf} 2$ ) are transcription factors that regulate tumor cell proliferation, transformation, survival, invasion, angiogenesis, metastasis, chemoresistance and radioresistance (16). In the current study, sequence analysis 


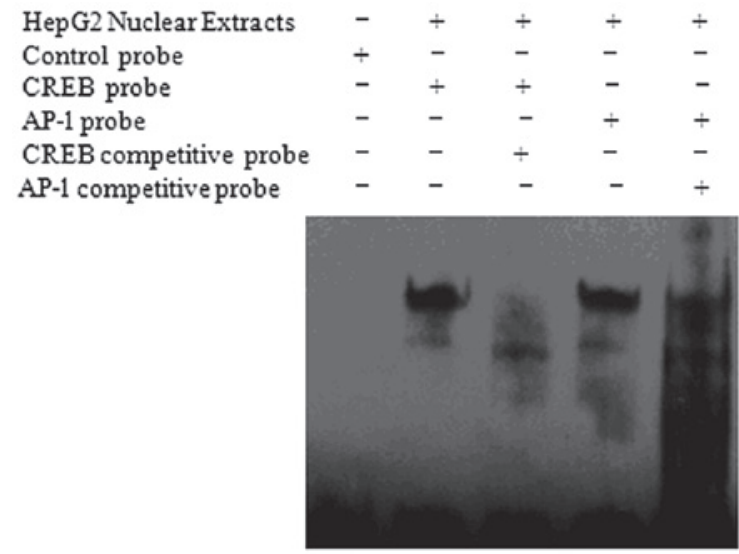

Figure 3. Binding activity of CREB and AP-1. From left to right, samples are control probe, CREB probe/nuclear extract, AP-1 probe/nuclear extract, CREB probe/nuclear extracts + competitive probe and AP-1 probe/nuclear extract + competitive probe. CREB and AP-1 bind with the promoter region. CREB binding was eliminated in the presence of the competitive probe, while AP-1 binding was partially blocked. CREB; cyclic-AMP response element binding; AP-1, activator protein-1.

identified numerous transcription factor binding sites within the predicted promoter sequence of TMEM174. Among these, CREB and AP-1 are involved in cell proliferation. EMSA indicated specific binding of CREB within the TMEM174 gene promoter region, while binding of the AP-1 was shown to be non-specific. Thus, CREB is suggested to be an important transcription factor involved in the regulation of TMEM174 gene expression, and the inhibition of CREB, and possibly AP-1, is suggested to inhibit the migration and invasion of cancer cells (17). Furthermore, cell line expression profiling showed that TMEM174 was expressed in the lymphadenoma-derived Raji cell line, indicating a role for TMEM174 in the development of lymphadenoma. CREB is overexpressed in the bone marrow of the majority of patients with acute myeloid leukemia (AML) and is associated with a poor initial outcome of clinical disease in AML patients. Moreover, CREB plays an important role in hematopoiesis (18-20). Thus, TMEM174 is suggested to play a role in leukemogenesis by activating CREB. Among the numerous transcription factors identified in the promoter sequence, CEBP has also been shown to be involved in inflammation (21). Therefore, TMEM174 is suggested to be involved in the regulation of pyelonephritis via CREB. However, these hypotheses require further investigation.

In conclusion, the results of the present study indicate that the core promoter region of the human TMEM174 gene is located in the region spanning -186 to $+674 \mathrm{bp}$ and that the transcription factors CREB and AP-1 are involved in the transcriptional regulation of this gene. These findings provide further insight into the mechanism of TMEM174 gene regulation, with the identification of CREB representing a basis for further studies concerning this mechanism.

\section{Acknowledgements}

This study was supported by the National Natural Science Foundation of China (nos. 81072093 and 30671092) and the Natural Science Foundation of Hebei Province (nos. C2009001260 and C2013209024).

\section{References}

1. Wan D, Gong Y, Qin W, et al: Large-scale cDNA transfection screening for genes related to cancer development and progression. Proc Natl Acad Sci USA 101: 15724-15729, 2004.

2. Wang P, Sun B, Hao D, Zhang X, Shi T and Ma D: Human TMEM174 that is highly expressed in kidney tissue activates AP-1 and promotes cell proliferation. Biochem Biophys Res Commun 394: 993-999, 2010.

3. Kinjo K, Sandoval S, Sakamoto KM and Shankar DB: The role of CREB as a proto-oncogene in hematopoiesis. Cell Cycle 4: 1134-1135, 2005.

4. Cho EC, Mitton B and Sakamoto KM: CREB and leukemogenesis. Crit Rev Oncog 16: 37-46, 2011.

5. De Falco V, Tamburrino A, Ventre S, et al: CD44 proteolysis increases CREB phosphorylation and sustains proliferation of thyroid cancer cells. Cancer Res 72: 1449-1458, 2012.

6. Mantamadiotis T, Papalexis N and Dworkin S: CREB signalling in neural stem/progenitor cells: recent developments and the implications for brain tumour biology. Bioessays 34: 293-300, 2012.

7. Dworkin S, Heath JK, deJong-Curtain TA, et al: CREB activity modulates neural cell proliferation, midbrain-hindbrain organization and patterning in zebrafish. Dev Biol 307: 127-141, 2007.

8. Yang C, Li X, Wang Y, Zhao L and Chen W: Long non-coding RNA UCA1 regulated cell cycle distribution via CREB through PI3-K dependent pathway in bladder carcinoma cells. Gene 496: 8-16, 2012.

9. Pearson G, Robinson F, Beers Gibson T, et al: Mitogen-activated protein (MAP) kinase pathways: regulation and physiological functions. Endocr Rev 22: 153-183, 2001.

10. Shen G, Jeong WS, Hu R and Kong AN: Regulation of Nrf2, NF-kappaB, and AP-1 signaling pathways by chemopreventive agents. Antioxid Redox Signal 7: 1648-1663, 2005.

11. Li G, Gustafson-Brown C, Hanks SK, et al: c-Jun is essential for organization of the epidermal leading edge. Dev Cell 4: 865-877, 2003.

12. Andrecht S, Kolbus A, Hartenstein B, Angel P and SchorppKistner M: Cell cycle promoting activity of JunB through cyclin A activation. J Biol Chem 277: 35961-35968, 2002.

13. Shaulian E and Karin M: AP-1 in cell proliferation and survival. Oncogene 20: 2390-2400, 2001.

14. Eferl R and Wagner EF: AP-1: a double-edged sword in tumorigenesis. Nat Rev Cancer 3: 859-868, 2003.

15. Weitzman JB, Fiette L, Matsuo K and Yaniv M: JunD protects cells from p53-dependent senescence and apoptosis. Mol Cell 6: 1109-1119, 2000.

16. Shanmugam MK, Nguyen AH, Kumar AP, Tan BK and Sethi G: Targeted inhibition of tumor proliferation, survival, and metastasis by pentacyclic triterpenoids: Potential role in prevention and therapy of cancer. Cancer Lett 320: 158-170, 2012.

17. Chien MH, Ying TH, Hsieh YS, et al: Dioscorea nipponica Makino inhibits migration and invasion of human oral cancer HSC-3 cells by transcriptional inhibition of matrix metalloproteinase-2 through modulation of CREB and AP-1 activity. Food Chem Toxicol 50: 558-566, 2012.

18. Shankar DB, Cheng JC and Sakamoto KM: Role of cyclic AMP response element binding protein in human leukemias. Cancer 104: 1819-1824, 2005.

19. Cheng JC, Esparza S, Sandoval S, Shankar D, Fu C and Sakamoto KM: Potential role of CREB as a prognostic marker in acute myeloid leukemia. Future Oncol 3: 475-480, 2007.

20. Shankar DB and Sakamoto KM: The role of cyclic-AMP binding protein (CREB) in leukemia cell proliferation and acute leukemias. Leuk Lymphoma 45: 265-270, 2004.

21. Söhle J, Machuy N, Smailbegovic E, et al: Identification of new genes involved in human adipogenesis and fat storage. PLoS One 7: e31193, 2012. 